\title{
Determinants of Pulmonary Blood Volume
}

\author{
Mitena L. Lewis, Julian Gnoj, Vincent J. Fisher, and \\ Lynn C. Christianson \\ From the Cardiopulmonary Laboratory, Medical Service, Veterans \\ Administration Hospital, 408 First Avenue, New York 10010
}

A B S T R A C T Pulmonary blood volume was determined by the radiocardiographic technique in 49 patients coming to cardiac catheterization. Since this method has not been directly compared with the more commonly used double injection of dye, 25 comparisons were carried out in 13 patients of the series. Agreement was good over a range of 4.5-21.1 heart cycles since there was no statistically significant difference between transit time values measured by the two methods.

The relation of pulmonary blood volume to other hemodynamic factors in these 49 patients, with and without cardiac or pulmonary disease, was evaluated by means of multiple regression analysis. The analysis carried out for mean transit time indicates that this parameter varies predominately with flow. Pulmonary blood volume, in this series of resting recumbent individuals, varies to a significant degree only with total blood volume and with pulmonary venous pressure. No parameters of vascular distensibility, such as pulmonary vascular resistance, were found to affect the volume of blood in the lungs.

The fact that variations in pulmonary blood volume among the subjects could be described by a multiple regression equation linear with respect to total blood volume and pulmonary venous pressure indicates that these variations are the result of passive distention of components of the vascular bed.

\section{INTRODUCTION}

Measurement of pulmonary blood volume has contributed to understanding of pulmonary hemodynamics. Numerous reports of data obtained by the injection of indicator into the pulmonary artery and left atrium are available in both patients with heart disease (1-10) and in normal subjects $(3,5,11,12)$. Nevertheless, there is

A preliminary report was published as an abstract in Clin. Res. 1967. 15: 346.

Received for publication 16 May 1969 and in revised form 4 August 1969. conflicting data on the relationship of lung blood volume to other hemodynamic factors, and hence the control of the size of this compartment is not clear, even in the resting state.

An alternative method of measuring pulmonary blood volume from precordial radioactive isotope dilution curves (radiocardiograms) (13) has been reported by Giuntini, Lewis, Sales Luis, and Harvey (14). The method is in use in this laboratory; and since only right heart catheterization is required, pulmonary blood volume measurements have been made in a wider variety of clinical states than usually described. The present report presents an analysis of this data which indicates that variations in pulmonary blood volume in all subjects studied, regardless of pathology, can be related to variations in only two factors, total blood volume and pulmonary venous pressure.

The radiocardiographic measurement of pulmonary blood volume is considered to have ill-defined anatomic limits by some authors (15) because the method is based on certain assumptions regarding the central circulation which cannot be evaluated in each subject. For this reason a comparison of the radiocardiographic and dye dilution methods in the same subjects is also included in this report to validate the statistical analysis of the data.

\section{METHODS}

Data from 57 consecutive cardiac catheterizations form the basis of the present report. Studies were not completed in eight patients; pertinent clinical data and diagnoses in the remaining 49 patients appear in Table $\mathrm{I}$. This group of 49 patients will be referred to as Group A. Data from a subsequent series of 21 patients, entitled Group B, are also included in this report for the purpose of assessing the accuracy of estimating equations for pulmonary blood volume derived from studies in Group A patients.

All patients were studied after an overnight fast. The volunteers, patients with cirrhosis, and patients with pulmonary disease were not sedated before the procedure. Other patients received $100 \mathrm{mg}$ of secobarbital $30 \mathrm{~min}$ before the procedure; in many cases $50 \mathrm{mg}$ of meperidine was also given intramuscularly. 
TABLE I

Clinical Data

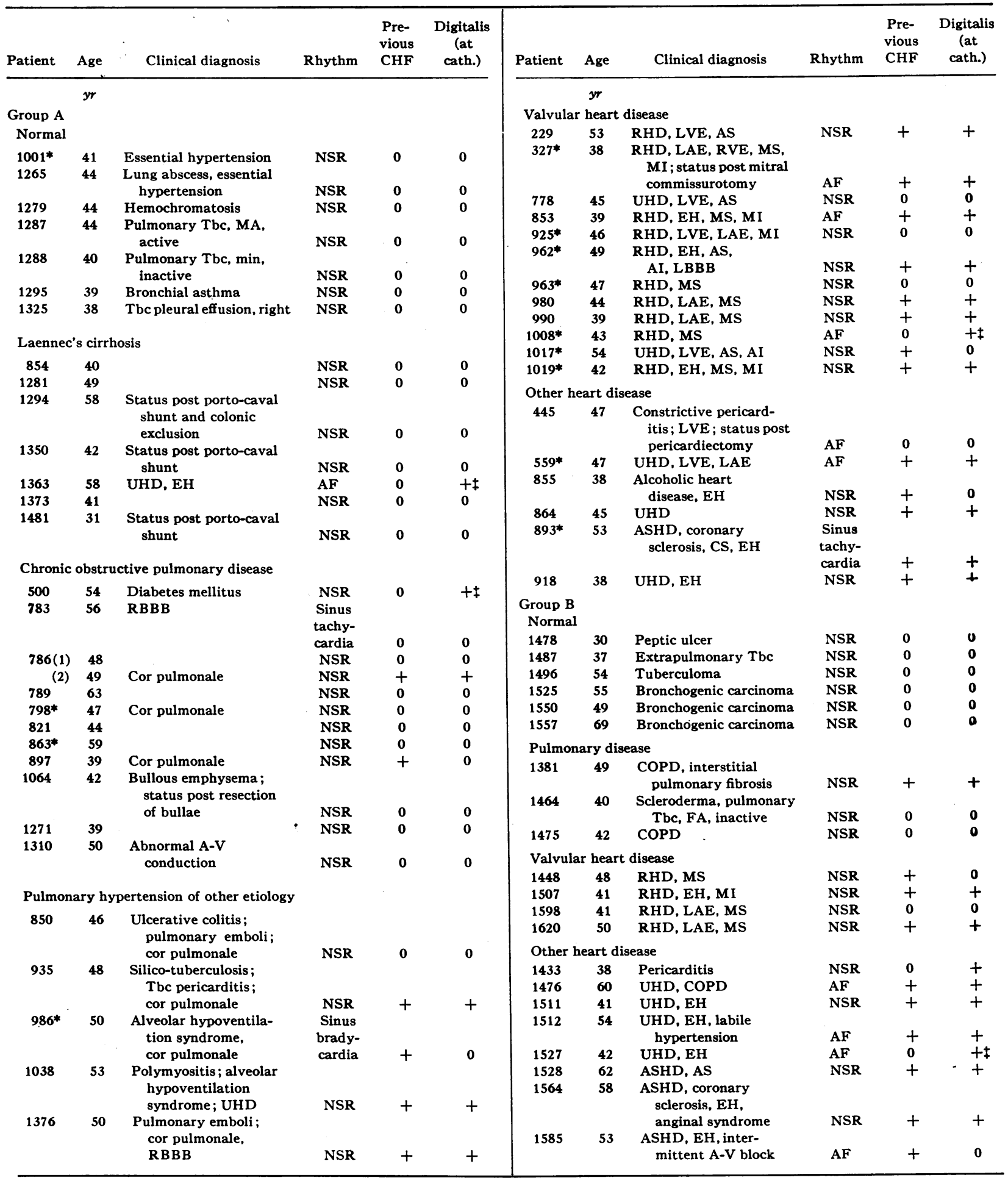

CHF, congestive heart failure; NSR, normal sinus rhythm; AF, atrial fibrillation; UHD, unknown heart disease; EH, enlarged heart; RBBB, right bundie branch block; RHD, rheumatic heart disease; LVE, left ventricular enlargement; $L A E$, left atrial enlargement; RVE, right ventricular enlarge-

* Patients in whom pulmonary mean transit time was measured both by radiocardiography and by double injection of dye.

$\neq$ Digitalis administered for control of cardiac rhythm. 
Pulmonary artery catheterization was carried out in the usual manner. In studies where the left atrium was entered, the Ross technique was used (16). The left ventricle was approached in retrograde fashion, via the femoral artery, by the Seldinger technique (17). For the injection of indicator a disposable radio-opaque polyvinyl catheter $(0.064$ in 0.D.) was introduced into the right atrium through an antecubital vein. An indwelling arterial needle was utilized for sampling of blood.

Pressures were recorded by means of Statham $P 23$ A transducers on a photographe recorder. ${ }^{1}$ Zero level for pressure readings was taken at $5 \mathrm{~cm}$ below the angle of Louis. A single fluid reservoir was connected to all pressure transducers and all amplifiers were adjusted to equal gain. The transducers were calibrated with a mercury manometer at the end of each catheterization. Systolic and diastolic pressures were read over two respiratory cycles and expressed as an average; mean pressure was obtained by planimetry of an electrically damped tracing during a similar period.

In 22 patients left atrial pressure was measured directly and in 20 others pulmonary "wedge" pressure was measured. In two patients, without mitral stenosis, left ventricular end diastolic pressure was used as a measure of pulmonary "venous" pressure. In the five remaining patients only pulmonary artery pressures were recorded. The diastolic pressures in these five patients ranged from 7 to $10 \mathrm{~mm}$ of $\mathrm{Hg}$. Since Kaltman, Herbert, Conroy, and Kossmann have reported that in this range pulmonary artery diastolic pressure varies little from left atrial pressure, these patients were included in the series (18).

The precordial dilution curves were recorded by means of a scintillation counter consisting of a $1.5 \times 1.0$ in $\mathrm{NaI}(\mathrm{Th})$ crystal and photomultiplier housed in a cylindrical lead collimator. ${ }^{2}$ The photomultiplier was connected to a ratemeter from which the output was fed into the photographic recorder. The injectate, radioiodinated human serum albumin (RISA), was diluted to contain $100 \mu \mathrm{Ci}$ of ${ }^{131} \mathrm{I} / \mathrm{ml}$. For each curve approximately $0.5 \mathrm{ml}$ was delivered via the right atrial catheter. Cornwall syringes ${ }^{3}$ were utilized for injection of indicator; injectates have shown consistent reproducibility in vivo and in vitro.

Cardiac output was determined from the radiocardiograms by the method of Donato, Rochester, Lewis, Durand, Parker, and Harvey (19). Typically, precordial isotope dilution curves present two activity peaks as tracer passes first through the right heart chambers and then through the left. Valid determinations of cardiac output may be obtained from such double curves if a calibration factor for the composite system of vascular sections is determined as described by Veall, Pearson, Hanley, and Lowe (20) and by Donato, Qiuntini, Lewis, Durand, Rochester, Harvey and Cournand (13).

Pulmonary mean transit time (PMTT) was calculated from the radiocardiograms as the difference between mean transit time of the right heart activity curve and the time of peak activity of the left heart curve. The theoretical background and method of analysis are discussed by Giun. tini et al. (14). Pulmonary blood volume (PBV) was obtained as the product of PMTT, measured in terms of heart rycles, and stroke volume (SV).

In 13 patients PMTT as measured from a radiocardiogram was compared with that derived from injection of indocyanine green dye into the pulmonary artery and left

\footnotetext{
${ }^{1}$ Electronics for Medicine, Inc., White Plains, N. Y.

Tracerlab, Waltham, Mass.

${ }^{3}$ Becton-Dickinson \& Co., Rutherford, N. J.
}

atrium. Approximately $2.5 \mathrm{mg}$ of dye was injected manually, first into the pulmonary artery catheter and subsequently through the Ross needle into the left atrium. The curves were recorded and analyzed in the usual manner $(21,22)$. Blood flow was determined from the pulmonary artery injection curves. Mean transit time through the pulmonary circulation was obtained as follows:

$$
\text { PMTT }=\text { MTT }_{\text {PA-D }}-\text { MTT }_{\text {LA-D }}
$$

where $\mathrm{MTT}_{\mathrm{PA}-\mathrm{D}}$ is the mean circulation time from the pulmonary artery to densitometer and $\mathrm{MTT}_{\mathbf{L A}-\mathrm{D}}$ is the mean circulation time from the left atrium to the densitometer.

In all patients total blood volume (TBV) was calculated from the dilution of RISA as determined from blood samples drawn before and after each injection. When several radiocardiograms were recorded, the increases in plasma counting rate after each injection were averaged $(\Delta C R)$ and plasma volume was obtained by the following:

$$
\text { Plasma volume }=\frac{\text { No. of counts injected }}{\Delta \mathrm{CR}}
$$

Total blood volume (TBV) was calculated from plasma volume and hematocrit (Hct) by the following:

$$
\mathrm{TBV}=\frac{\text { Plasma volume }}{1-\mathrm{Hct}}
$$

Hematocrits were corrected for trapped plasma according to the graph of Chaplin and Mollison (23). Circulating blood hematocrit was converted to total body hematocrit using a factor of 0.896 (24).

In 29 patients TBV was also determined with T-1824 dye. 2-4 $\mathrm{ml}$ of undiluted dye were injected from a Cornwall syringe into the pulmonary artery catheter which was then flushed with $10 \mathrm{ml}$ of saline. Samples of arterial blood obtained at known intervals of time after injection were analyzed for plasma concentration of dye in a Beckman DU spectrophotometer at $630 \mathrm{~m} \mu$. These concentrations were plotted against time on semilogarithmic paper and the curve extrapolated to obtain the concentration at zero time, $\mathrm{C}_{0}$. Plasma volume was calculated as follows:

$$
\text { Plasma volume }=\frac{\text { milligrams of dye injected }}{\mathrm{C}_{0}}
$$

Total blood volume was derived from plasma volume and hematocrit as indicated above.

The principal texts used as an aid in the subsequent statistical analysis were those of Steel and Torrie (25) and of Ezekiel and Fox (26)

\section{RESULTS}

Validation of method. The overall success rate of the radiocardiographic method was $86 \%$. In four patients poor definition of the right and left heart activity curves resulted from severe reduction in blood flow (arteriovenous oxygen difference range 9.0-14.9 volume per cent). In four other patients in whom cardiac output was not severely impaired (arteriovenous oxygen difference range 4.6-7.3 volumes per cent), inadequate radiocardiograms were the result of faulty collimation. These eight studies were excluded from the series. Hemodynamic data from the remaining 49 and from all subjects in Group B appear in Table II. 
Comparison of PMTT measured by the double injection of dye and by radiocardiography is presented in Fig. 1. 25 determinations were made in the course of 13 cardiac catheterizations. The patients in whom these comparisons were made are indicated by an asterisk in Table I. The regression equation for PMTT, expressed in heart cycles (HC), is the following:

$$
\begin{aligned}
\operatorname{PMTT}_{\text {RISA }}(\mathrm{HC})= & 0.1235 \\
& +1.0988 \operatorname{PMTT}_{\text {Dye }}(\mathrm{HC})
\end{aligned}
$$

The correlation coefficient is 0.942 ; the standard error of estimate $s_{y} . x$ is $1.86 \mathrm{HC}$. The regression line and line of identity are not significantly different from each other $(t=1.208$, df $23,0.3>P>0.2)$. A similar analysis of the data, expressed in terms of seconds rather than heart cycles, results in a correlation coefficient of $0.855 ; \mathrm{s}_{y} .=1.57 \mathrm{sec}$. Agreement is better when measured in heart cycles since some discrepancies due to variations in heart rate are eliminated.

Reproducibility of the radiocardiographic method was evaluated by subtracting the value of the second PMTT measurement from that of the first. These differences were averaged to obtain the mean value, $\bar{\Delta}$, and the standard deviation of differences about this mean, $\mathbf{S D}_{\Delta}$, was calculated. Duplicate determinations of PMTT PrsA were available on 34 patients (Fig. 2). The mean difference for duplicate PMTT RIss determinations was 0.2 $\pm 0.8 \mathrm{HC}$, not significantly different from zero $(t=$ 1.631, $0.2>P>0.1$ ). Expressed as per cent of the mean of duplicate values, SDs was $9 \%$ for PMTTRIss and $11 \%$ for $\mathrm{PBV}_{\mathrm{RISA}}$, indicating that in all probability two determinations which differ by more than $20 \%$ represent a true change in the volume of blood in the lungs.

19 comparisons of cardiac output measured by radiocardiography and by dye dilution are presented in Fig. 3. The mean difference between $Q_{\text {rIsA }}$ and $Q_{\text {Drw }}$ was not significantly different from zero $(\bar{\Delta}=63 \mathrm{ml} \pm 718 \mathrm{ml} ; t=$ $0.38, P>0.8$ ). Reproducibility of both methods was also good. The mean difference between duplicate determinations was not significantly different from zero for either method; SDs was $10 \%$ of the mean of duplicates for $Q_{\text {risa }}$ and $7 \%$ for $Q_{\text {nye. }}$

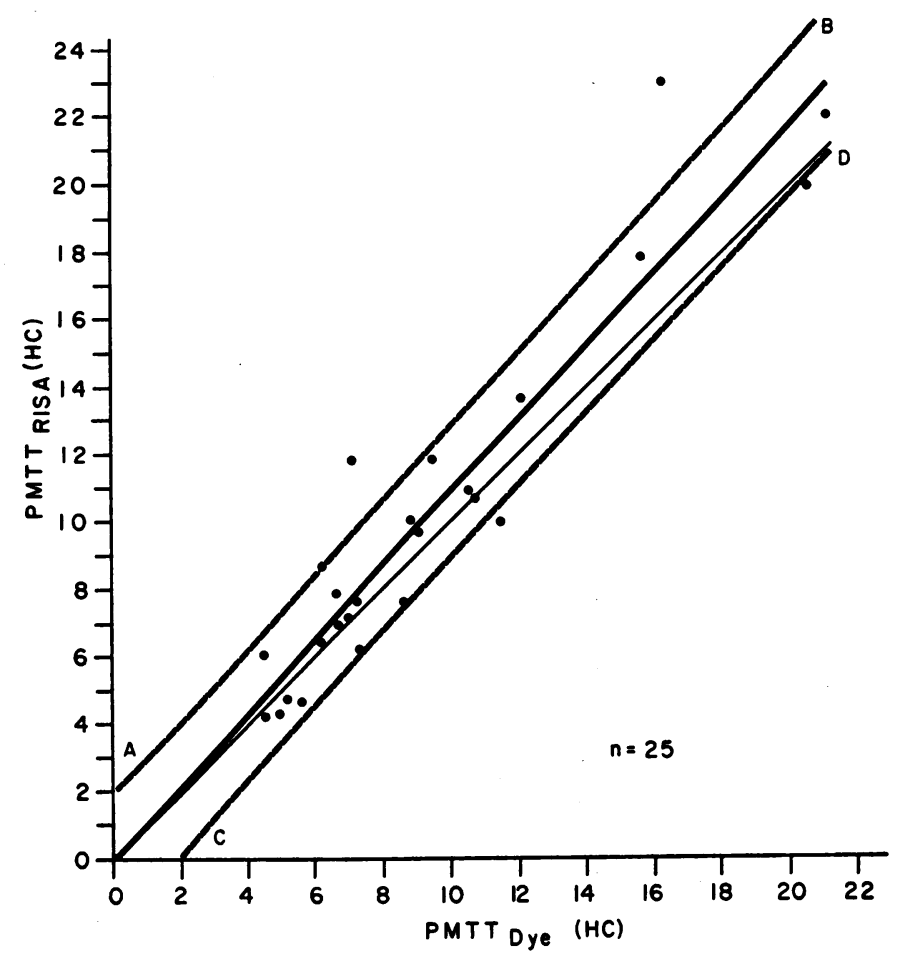

FIGURE 1 Comparison of pulmonary mean transit time (PMTT) values determined by two indicator dilution techniques. PMTT PIss $_{\text {A }}$ $=$ transit time determined from precordial radiocardiograms. PMTT $_{\text {Dye }}=$ transit time determined from the injection of indocyanine green into pulmonary artery and left atrium. $\mathrm{HC}=$ heart cycle. 25 determinations in 13 patients are presented. The fine solid line is the line of identity. The heavy solid line is the regression line and the broken lines, $A B$ and $C D$, represent one standard error of estimate of the regression. 
TABLE II

Hemodynamic Data

\begin{tabular}{|c|c|c|c|c|c|c|c|c|c|c|c|c|c|}
\hline $\begin{array}{c}\text { Patient } \\
\text { No. }\end{array}$ & BSA & c.o. & HR & sV & $\mathbf{P M}$ & & PBV & PBV & $\mathbf{P}_{\mathbf{P A}}$ & $\mathbf{P L}_{\mathbf{L}}$ & $\mathbf{P}_{\mathbf{B A}}$ & PVR & TBV \\
\hline \multicolumn{14}{|l|}{ Group A } \\
\hline \multicolumn{14}{|l|}{ Normal } \\
\hline 1001 & 1.88 & 5935 & 84 & 71 & 6.1 & 4.4 & 433 & 230 & $20 / 7(13)$ & $(8)^{*}$ & $160 / 102(118)$ & 1.6 & 3948 \\
\hline 1265 & 1.63 & 5462 & 78 & 70 & 6.5 & 5.0 & 455 & 279 & $13 / 7(9)$ & (6) & $159 / 96(122)$ & 0.9 & 4709 \\
\hline 1279 & 1.64 & 5577 & 79 & 71 & 5.6 & 4.3 & 398 & 243 & $18 / 9(13)$ & (5) & $132 / 77(100)$ & 2.4 & 6073 \\
\hline 1287 & 2.05 & 5322 & 70 & 76 & 5.5 & 4.7 & 418 & 204 & $24 / 14(19)$ & (4) & $122 / 72(94)$ & 5.8 & 4479 \\
\hline 1288 & 2.15 & 8947 & 75 & 119 & 5.6 & 4.5 & 666 & 310 & $25 / 13(16)$ & (8) & $122 / 67(89)$ & 1.9 & 5891 \\
\hline 1295 & 1.69 & 5329 & 71 & 75 & 5.2 & 4.4 & 390 & 231 & $16 / 7(10)$ & (6) & $123 / 75(96)$ & 1.3 & 4243 \\
\hline 1325 & 1.68 & 7371 & 83 & 89 & 5.4 & 3.9 & 481 & 286 & $14 / 7(10)$ & $7 \ddagger$ & $116 / 62(87)$ & 0.7 & 5374 \\
\hline \multicolumn{14}{|c|}{ Laennec's cirrhosis } \\
\hline 854 & 1.88 & 11982 & 100 & 120 & 4.1 & 2.5 & 492 & 262 & $22 / 10(15)$ & $10 \ddagger$ & $116 / 68(85)$ & 0.8 & 7451 \\
\hline 1281 & 2.14 & 7468 & 69 & 108 & 6.0 & 5.3 & 648 & 303 & $18 / 7(11)$ & $7 \ddagger$ & $114 / 66(85)$ & 1.2 & 7034 \\
\hline 1294 & 2.05 & 7481 & 72 & 104 & 5.3 & 4.4 & 551 & 269 & $54 / 22(36)$ & (15) & $187 / 78(127)$ & 5.8 & 5037 \\
\hline 1350 & 2.10 & 9439 & 92 & 103 & 5.7 & 3.7 & 587 & 280 & $27 / 7(12)$ & $7 \ddagger$ & - & 1.1 & 5710 \\
\hline 1363 & 1.66 & 5069 & 95 & 53 & 9.2 & 5.8 & 488 & 294 & $24 / 11(17)$ & (11) & $126 / 66(89)$ & 2.0 & 4736 \\
\hline 1373 & 1.82 & 9044 & 95 & 95 & 6.6 & 4.2 & 627 & 345 & $19 / 6(11)$ & (5) & $132 / 68(91)$ & 1.2 & 5503 \\
\hline 1481 & 2.06 & 11293 & 87 & 130 & 5.8 & 4.0 & 754 & 366 & $18 / 9(12)$ & (8) & - & 0.7 & 6555 \\
\hline \multicolumn{14}{|c|}{ Chronic obstructive pulmonary disease } \\
\hline 500 & 1.88 & 4823 & 72 & 67 & 5.5 & 4.5 & 369 & 196 & $26 / 11(17)$ & $(8)^{*}$ & $118 / 58(81)$ & 3.5 & 4048 \\
\hline 783 & 1.70 & 4474 & 112 & 40 & 10.0 & 5.3 & 400 & 235 & $24 / 9(16)$ & 97 & - & 2.7 & 4144 \\
\hline $786(1)$ & 1.59 & 5920 & 96 & 62 & 6.6 & 4.1 & 409 & 257 & $23 / 11(15)$ & (8) & $116 / 65(83)$ & 1.9 & 3960 \\
\hline (2) & 1.46 & 7757 & 109 & 71 & 10.6 & 6.1 & 753 & 516 & $52 / 4 \mathrm{RV}$ & $1 \S$ & $161 / 77(107)$ & - & 3605 \\
\hline 789 & 1.69 & 7963 & 81 & 98 & 7.4 & 5.8 & 725 & 429 & $25 / 10(14)$ & $10 \ddagger$ & $132 / 64(88)$ & 0.9 & 6254 \\
\hline 798 & 1.50 & 5972 & 76 & 79 & 7.6 & 6.0 & 600 & 400 & $23 / 8(14)$ & $(2)^{*}$ & $112 / 64(75)$ & 3.0 & 4732 \\
\hline 821 & 1.86 & 7113 & 75 & 95 & 6.1 & 4.9 & 580 & 313 & $23 / 9(13)$ & $9 \ddagger$ & $109 / 63(83)$ & 1.0 & 5527 \\
\hline 863 & 1.64 & 7862 & 98 & 80 & 10.1 & 6.2 & 808 & 493 & $33 / 16(20)$ & $(5)^{*}$ & $124 / 79(96)$ & 3.1 & 5890 \\
\hline 897 & 2.18 & 7625 & 98 & 78 & 7.5 & 4.6 & 585 & 268 & $33 / 14(23)$ & $(1)^{*}$ & $144 / 72(94)$ & 6.3 & 6682 \\
\hline 1064 & 1.86 & 5820 & 95 & 61 & 6.5 & 4.1 & 399 & 213 & $27 / 15(20)$ & (5) & - & 4.8 & 5477 \\
\hline 1271 & 1.96 & 5453 & 81 & 67 & 4.9 & 3.6 & 328 & 167 & $35 / 16(23)$ & (12) & $138 / 72(100)$ & 4.0 & 5128 \\
\hline 1310 & 1.74 & 4923 & 64 & 77 & 5.7 & 5.3 & 439 & 252 & $17 / 6(10)$ & $6 \ddagger$ & $158 / 89(116)$ & 1.4 & 5151 \\
\hline \multicolumn{14}{|c|}{ Pulmonary hypertension of other etiology } \\
\hline 850 & 1.84 & 6589 & 81 & 81 & 7.9 & 5.9 & 640 & 348 & $98 / 38(57)$ & (12) & $127 / 71(93)$ & 12.6 & 5166 \\
\hline 935 & 1.66 & 5003 & 91 & 55 & 7.1 & 4.7 & 391 & 236 & $72 / 19(37)$ & (3) & $131 / 75(97)$ & 11.3 & 5818 \\
\hline 986 & 1.98 & 7078 & 47 & 151 & 4.4 & 5.5 & 664 & 335 & $57 / 24(33)$ & (9) & $147 / 70(98)$ & 6.7 & 5235 \\
\hline 1038 & 2.20 & 8247 & 91 & 91 & 6.7 & 4.4 & 610 & 277 & $34 / 13(22)$ & $(10)$ & $138 / 82(91)$ & 3.2 & 6348 \\
\hline 1376 & 1.79 & 4650 & 67 & 69 & 9.8 & 8.9 & 676 & 378 & $85 / 28(48)$ & $(3)^{*}$ & - & 16.7 & 5266 \\
\hline \multicolumn{14}{|c|}{ Valvular heart disease } \\
\hline 229 & 1.97 & 5054 & 76 & 66 & 11.6 & 9.2 & 766 & 389 & $46 / 23(31)$ & $(24)^{*}$ & $97 / 64(77)$ & 2.6 & 5915 \\
\hline 327 & 1.69 & 4036 & 69 & 59 & 11.8 & 10.4 & 696 & 412 & $31 / 14(24)$ & $(15)^{*}$ & $107 / 64(74)$ & 3.4 & 5041 \\
\hline 778 & 1.75 & 6900 & 76 & 91 & 9.0 & 7.0 & 819 & 468 & 一 & $(19)^{*}$ & $89 / 68(76)$ & - & 6191 \\
\hline 853 & 2.10 & 5779 & 75 & 80 & 11.7 & 9.7 & 936 & 446 & $47 / 25(32)$ & $(22)^{*}$ & $139 / 71(86)$ & 3.6 & 6104 \\
\hline
\end{tabular}

C.O., cardiac output; HR, heart rate in beats per minute; SV, stroke volume; PMTT, pulmonary mean transit time ; HC, heart cycle; $\mathrm{PBV}$, pulmonary blood volume; $\mathrm{P}_{\mathrm{PA}}$, pulmonary artery pressure; $\mathrm{P}_{\mathrm{L}}$, pulmonary venous pressure, measured as pulmonary "wedge" pressure unless otherwise specified; $P_{\mathbf{B A}}$, brachial arterial pressure; PVR, pulmonary vascular resistance; RU, resistance units defined as gradient between pulmonary arterial and left atrial mean pressures (in $\mathrm{mm} \mathrm{Hg}$ ), divided by cardiac output (in liters/min per $\mathrm{m}^{2} \mathrm{BSA}$ ); $\mathrm{TBV}$, total blood volume.

* Left atrial pressure.

‡ Pulmonary arterial diastolic pressure.

$\S$ Left ventricular end diastolic pressure. 
TABLE II (Continued)

\begin{tabular}{|c|c|c|c|c|c|c|c|c|c|c|c|c|c|}
\hline \multirow[t]{2}{*}{$\begin{array}{l}\text { Patient } \\
\text { No. }\end{array}$} & \multirow{2}{*}{$\frac{\text { BSA }}{m^{2}}$} & \multirow{2}{*}{$\frac{\text { c.o. }}{m l / m i n}$} & \multirow[t]{2}{*}{ HR } & \multirow{2}{*}{$\frac{\mathrm{SV}}{m l}$} & \multicolumn{2}{|c|}{ PMTT } & \multirow{2}{*}{$\frac{\text { PBV }}{m l}$} & \multirow{2}{*}{$\frac{\mathrm{PBV}}{\mathrm{ml} / \mathrm{m}^{2}}$} & \multirow{2}{*}{$\frac{\mathrm{PPA}_{\mathrm{A}}}{m m \mathrm{Hg}_{\mathrm{g}}}$} & \multirow{2}{*}{$\frac{\mathrm{PL}_{\mathrm{L}}}{m m \mathrm{Hg}}$} & \multirow{2}{*}{$\frac{\mathrm{P}_{\mathrm{BA}}}{\mathrm{mm \textrm {Hg }}}$} & \multirow{2}{*}{$\begin{array}{c}\text { PVR } \\
R U\end{array}$} & \multirow{2}{*}{$\frac{\mathrm{TBV}}{m l}$} \\
\hline & & & & & $H C$ & sec & & & & & & & \\
\hline \multicolumn{14}{|c|}{ Valvular heart disease (Continued) } \\
\hline 925 & 1.66 & 4291 & 82 & 52 & 10.7 & 7.9 & 556 & 335 & $50 / 18(28)$ & 198 & $120 / 66(94)$ & 3.5 & 4119 \\
\hline 962 & 1.58 & 3055 & 83 & 38 & 17.9 & 13.0 & 680 & 430 & $76 / 32(47)$ & (29)* & $135 / 51(78)$ & 9.3 & 4793 \\
\hline 963 & 1.86 & 4385 & 63 & 70 & 6.9 & 6.6 & 483 & 260 & $24 / 10(12)$ & $(8)^{*}$ & $115 / 64(85)$ & 1.7 & 3660 \\
\hline 980 & 2.00 & 5121 & 60 & 85 & 7.2 & 7.2 & 612 & 306 & $27 / 11(14)$ & (11) & $115 / 61(78)$ & 3.5 & 5475 \\
\hline 990 & 1.56 & 3226 & 69 & 47 & 9.9 & 8.5 & 465 & 298 & - & (28)* & $121 / 75(97)$ & - & 3200 \\
\hline 1008 & 2.04 & 4717 & 73 & 65 & 11.8 & 9.7 & 767 & 376 & $32 / 15(18)$ & (11)* & $104 / 63(71)$ & 3.0 & 5461 \\
\hline 1017 & 1.78 & 4609 & 58 & 79 & 6.0 & 6.2 & 474 & 266 & $24 / 10(13)$ & (10)* & $148 / 73(95)$ & 1.2 & 4150 \\
\hline 1019 & 2.13 & 4368 & 116 & 38 & 23.1 & 12.0 & 878 & 412 & $104 / 47(61)$ & $(35)^{*}$ & $125 / 91(102)$ & 12.7 & 6867 \\
\hline \multicolumn{14}{|c|}{ Other heart disease } \\
\hline 445 & 1.86 & 4042 & 77 & 53 & 8.2 & 6.4 & 435 & 234 & $25 / 9(15)$ & $(9)^{*}$ & - & 2.8 & 5166 \\
\hline 559 & 1.80 & 5220 & 51 & 102 & 7.8 & 9.1 & 796 & 442 & $24 / 12(17)$ & $(12)^{*}$ & $101 / 50(65)$ & 1.7 & 5222 \\
\hline 855 & 1.94 & 8855 & 90 & 98 & 5.8 & 3.9 & 568 & 293 & $12 / 4(7)$ & (3)* & $138 / 63(87)$ & 0.9 & 6047 \\
\hline 864 & 1.86 & 5720 & 92 & 62 & 10.4 & 6.7 & 645 & 347 & $33 / 17(22)$ & (10)* & $174 / 89$ & 3.9 & 5118 \\
\hline 893 & 1.77 & 3724 & 90 & 41 & 14.3 & 9.6 & 586 & 331 & $23 / 10(14)$ & $(2)^{*}$ & $120 / 71(83)$ & 5.7 & 4526 \\
\hline 918 & 1.94 & 3580 & 77 & 47 & 13.5 & 10.6 & 635 & 327 & $22 / 10(14)$ & $(4)^{*}$ & $101 / 68(79)$ & 5.4 & 5050 \\
\hline \multicolumn{14}{|l|}{ Group B } \\
\hline \multicolumn{14}{|c|}{ Normal } \\
\hline 1478 & 1.79 & 5685 & 55 & 103 & 6.2 & 6.8 & 639 & 357 & $22 / 10(15)$ & $10 \ddagger$ & $124 / 74(94)$ & 1.6 & 4847 \\
\hline 1487 & 1.55 & 6300 & 91 & 69 & 6.0 & 3.8 & 414 & 267 & $22 / 14(18)$ & (10) & $128 / 97(110)$ & 2.0 & 3892 \\
\hline 1496 & 1.64 & 8042 & 79 & 102 & 4.9 & 3.7 & 500 & 305 & $29 / 10(18)$ & (9) & $137 / 22(101)$ & 1.8 & 3969 \\
\hline 1525 & 2.04 & 7063 & 89 & 79 & 5.2 & 3.5 & 411 & 201 & $26 / 12(16)$ & (6) & $151 / 85(112)$ & 2.9 & 5960 \\
\hline 1550 & 2.02 & 9981 & 72 & 139 & 4.9 & 4.1 & 681 & 337 & $20 / 6(11)$ & (2) & $154 / 83(112)$ & 1.8 & 5266 \\
\hline 1557 & 1.83 & 6399 & 78 & 82 & 5.7 & 4.4 & 467 & 255 & $21 / 8(14)$ & (6) & $161 / 64(103)$ & 2.3 & 4862 \\
\hline \multicolumn{14}{|c|}{ Pulmonary disease } \\
\hline 1381 & 1.64 & 3869 & 97 & 40 & 8.9 & 5.5 & 356 & 217 & $86 / 44(59)$ & (11) & $119 / 66(81)$ & 20.4 & 5021 \\
\hline 1464 & 1.93 & 5622 & 93 & 60 & 4.4 & 2.9 & 264 & 137 & $38 / 13(22)$ & $(0)$ & $124 / 71(95)$ & 7.6 & 5324 \\
\hline 1475 & 2.22 & 7420 & 90 & 82 & 4.1 & 2.7 & 336 & 151 & $24 / 10(16)$ & (5) & $137 / 81(97)$ & 3.3 & 5449 \\
\hline \multicolumn{14}{|c|}{ Valvular heart disease } \\
\hline 1448 & 1.72 & 4814 & 65 & 74 & 8.2 & 7.6 & 607 & 353 & $25 / 8(15)$ & (7) & $119 / 66(88)$ & 2.9 & 4482 \\
\hline 1507 & 1.64 & 3772 & 114 & 33 & 18.8 & 9.9 & 620 & 378 & $74 / 33(42)$ & (33) & $111 / 77(86)$ & 3.9 & 4808 \\
\hline 1598 & 1.66 & 5117 & 73 & 70 & 7.5 & 6.1 & 525 & 316 & $36 / 13(21)$ & (13) & $112 / 56(73)$ & 2.6 & 4183 \\
\hline 1620 & 1.67 & 6767 & 64 & 106 & 6.6 & 6.2 & 700 & 419 & $59 / 23(36)$ & (21) & $118 / 55(75)$ & 3.7 & 5080 \\
\hline \multicolumn{14}{|c|}{ Other heart disease } \\
\hline 1433 & 1.95 & 9003 & 73 & 123 & 5.8 & 4.8 & 713 & 366 & $20 / 7(11)$ & (7) & $121 / 72(94)$ & 0.9 & 4666 \\
\hline 1476 & 1.80 & 3524 & 88 & 40 & 11.1 & 7.6 & 444 & 247 & $24 / 10(15)$ & (9) & $111 / 63(83)$ & 3.1 & 4106 \\
\hline 1511 & 2.20 & 4295 & 99 & 43 & 18.2 & 11.1 & 783 & 356 & $47 / 30(37)$ & (29) & $102 / 65(80)$ & 4.1 & 6324 \\
\hline 1512 & 1.83 & 4867 & 77 & 63 & 10.1 & 7.9 & 636 & 348 & $42 / 17(26)$ & (12) & $193 / 116(136)$ & 5.3 & 5217 \\
\hline 1527 & 1.84 & 2770 & 67 & 41 & 9.8 & 8.8 & 402 & 218 & $32 / 13(19)$ & (9) & $160 / 93(114)$ & 6.6 & 4206 \\
\hline 1528 & 1.98 & 5250 & 74 & 71 & 7.2 & 5.8 & 511 & 258 & $28 / 14(17)$ & (6) & $153 / 77(105)$ & 4.1 & 5169 \\
\hline 1564 & 2.04 & 5700 & 58 & 98 & 7.9 & 8.2 & 774 & 379 & $30 / 13(18)$ & (12) & $155 / 67$ (94) & 2.1 & 5299 \\
\hline 1585 & 1.87 & 5543 & 67 & 83 & 6.7 & 6.0 & 556 & 297 & $48 / 19(30)$ & (19) & $203 / 79(115)$ & 3.7 & 5100 \\
\hline
\end{tabular}

Total blood volume measured by dilution of RISA is not significantly different from zero $(t=0.613, P>$ was compared with values obtained by dilution of $T-1824$ dye in 22 patients. The mean difference between TBV $V_{\text {Dye }}$ and $T B V_{\text {RIBA }}$ was $46 \pm 354 \mathrm{ml} / \mathrm{m}^{2}$ BSA which $0.50)$.

Determinants of pulmonary blood volume. A multiple regression analysis was used to identify the determinants 
of pulmonary blood volume. Since PBV is not measured directly but is calculated from transit time and flow measurements, analysis was first carried out for PMTT. The independent variables included in the multiple analysis are those showing a significant correlation to PMTT and PBV in simple linear regression equation listed in Table III. The data for cardiac output, PMTT, and TBV are those derived from injection of RISA. Where duplicate radiocardiograms were recorded, only data from the first curve were used in the statistical analysis.

A highly significant estimating equation for pulmonary mean transit time in the resting recumbent individual was calculated from the data as follows:

$$
\operatorname{PMTT}(\mathrm{HC})=\frac{6.658 \mathrm{P}_{\mathrm{L}}+0.097 \mathrm{TBV}}{\mathrm{SV}}
$$

This equation includes only three independent variables, stroke volume, pulmonary "venous" pressure, and total blood volume, and passes through the origin; regression coefficient $(R)=0.980$; the standard error of estimate is 1.82 heart cycles.

Using this estimating equation for PMTT and the hemodynamic data obtained in the 21 patients of Group $\mathrm{B}$, the observed and predicted values of PMTT for this group of patients are compared in Fig. 4.16 of the 21 comparisons fall within one standard error of estimate

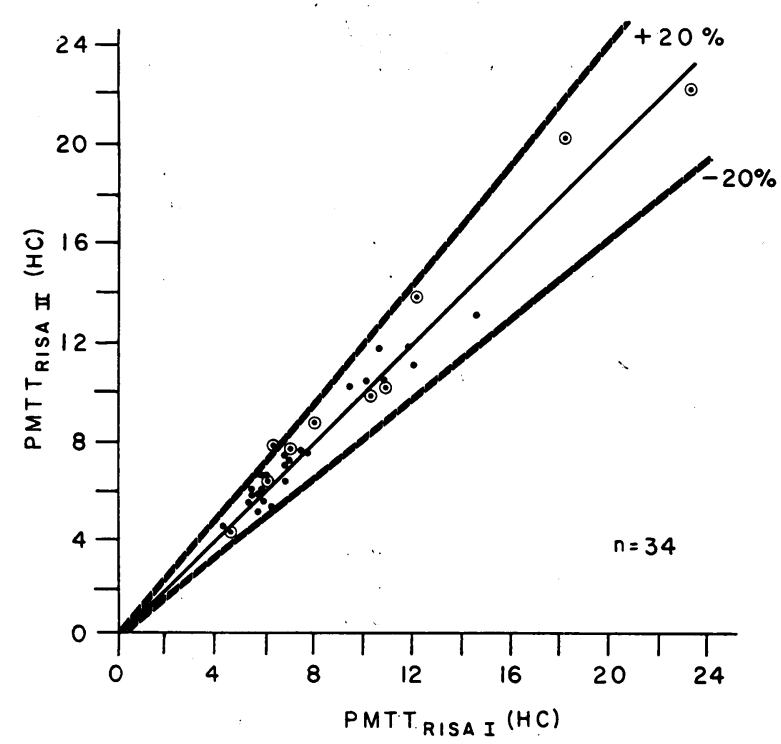

Figure 2 Reproducibility of PMTT RISA $_{\text {in }} 34$ patients. The circled points represent data from patients in whom PMTT $_{\text {Dye }}$ studies were also made.

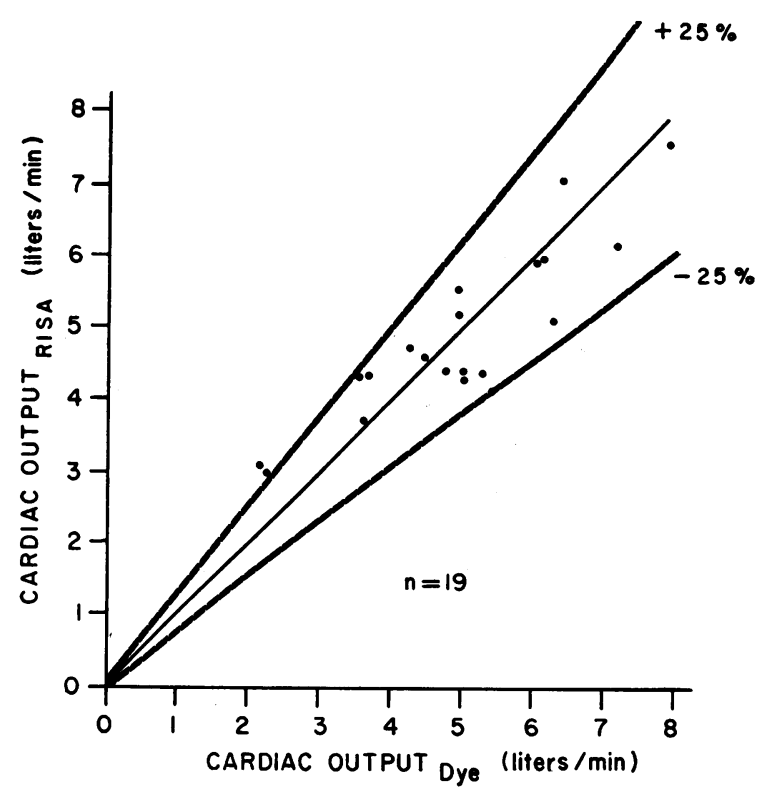

FIgURE 3 Comparison of cardiac output values obtained from radiocardiograms, and from injection of indocyanine green dye into the pulmonary artery. 19 determinations in 13 patients are presented. The solid line is the line of identity.

and all but two points within two standard errors of the line of identity.

The form of equation 6 derives from the observation, illustrated in Fig. 5, that PMTT bears an inverse relationship to stroke volume as follows:

$$
\mathrm{PMTT}=\frac{K}{\mathrm{SV}}
$$

This implies that pulmonary blood volume, the product of PMTT and stroke volume, is a constant $(K)$. However, such a conclusion is not substantiated by the data in Group A patients (Table II). Alternatively, the points in Fig. 5 might be fitted by a family of hyperbolic regression lines each with a different value of $K$ or $P B V$. The individual values of $K$ might be the result of variation in some other hemodynamic factor. To evaluate this possibility multiple regression equations were constructed maintaining the hyperbolic form, and the following was found to explain the largest fraction of the variance of PMTT, i.e., $75 \%$.

$$
\begin{aligned}
\mathrm{PMTT}=0.802 & +7.249\left(\mathrm{P}_{\mathrm{L}} / \mathrm{SV}\right) \\
& +0.087(\mathrm{TBV} / \mathrm{SV}) \quad \mathrm{R}=\mathbf{0 . 8 6 7}
\end{aligned}
$$

The significance of both these ratios, $\mathrm{P}_{\mathrm{L}} / \mathrm{SV}$ and $\mathrm{TBV} / \mathrm{SV}$, in the multiple regression equation was established by analysis of variance as seen in the ac- 
companying table based on equation 8:

Tabulation (based on Equation 8)

\begin{tabular}{lrrrr}
\hline \multicolumn{1}{c}{ Source } & df & $\begin{array}{c}\text { Sum of } \\
\text { squares }\end{array}$ & $\begin{array}{r}\text { Mean } \\
\text { square }\end{array}$ & \multicolumn{1}{c}{$\boldsymbol{F}$} \\
\hline Total & 48 & 618.56 & & \\
Reg on TBV/SV & 1 & 299.48 & 299.48 & \\
Residual & 47 & 319.08 & 6.79 & 44.11 \\
Reg on $\mathrm{P}_{\mathrm{L}} / \mathrm{SV}$ & 1 & 165.42 & 165.42 & \\
Residual & 46 & 153.66 & 3.34 & 49.53 \\
\hline
\end{tabular}

Other parameters, specifically pulmonary artery systolic pressures ( $\left.P_{P \Delta s}\right)$, mean intravascular pressure (MIP), pulmonary vascular resistance ( $P V R)$, and the gradient between pulmonary artery diastolic and left atrial mean pressure $(\Delta \mathrm{P})$ do not contribute significantly to an explanation of the variance of PMTT when used in multiple regression equations involving three independent variables.

The simplification of equation 8 to equation 6 , which has the form of a rectangular hyperbola, is justified since the $\mathrm{Y}$ intercept of equation $8(0.802)$ falls within one standard error of estimate of the regression line (1.8 heart cycles). Hence the $\mathrm{Y}$ intercept may in fact be zero. Equation 6 was obtained by the method of least squares assuming that the regression line passes through the origin rather than the mean. Analysis of variance indicates that the reduction of unexplained variance of PMTT to be gained by fitting the regression to the mean (equation 8$)$ is not significant $(F=0.581)$.

By a simple mathematical transposition, equation 6 becames an estimating equation for pulmonary blood volume as follows:

$$
(\mathrm{PMTT} \times \mathrm{SV})=6.658 \mathrm{P}_{\mathrm{L}}+0.097 \mathrm{TBV}
$$

This equation includes only two independent variables. The regression coefficient $(R)$; obtained by multiplying the sum of square for each independent variable by the partial regression coefficient for that variable, is 0.950 (25). The standard error of estimate is $118 \mathrm{ml}$.

TABLE III

Regression Equations Relating Pulmonary Mean Transit Time and Pulmonary Blood Volume to Other Hemodynamic Parameters*

\begin{tabular}{|c|c|c|c|c|c|c|}
\hline $\mathbf{N}$ & Parameter & Regression equation & $S_{b}$ & $\mathbf{r}$ & $t_{\mathbf{r}}$ & $\mathbf{P r}_{\mathbf{r}}$ \\
\hline \multicolumn{7}{|c|}{ Pulmonary mean transit time } \\
\hline 49 & SV & $Y=15.39-0.09 X$ & 0.01 & 0.629 & 5.566 & $<0.001$ \\
\hline 49 & $1 / \mathrm{SV}$ & $Y=0.13+567 X$ & 22.62 & 0.753 & 7.845 & $<0.001$ \\
\hline 49 & $\mathrm{P}_{\mathrm{L}}$ & $Y=5.41+0.29 X$ & 0.06 & 0.592 & 5.060 & $<0.001$ \\
\hline 49 & TBV & $Y=8.40-0.03 X$ & 0.55 & 0.015 & 0.103 & $>0.90$ \\
\hline 49 & $\mathrm{TBV} / \mathrm{SV}$ & $Y=0.60+0.12 X$ & 0.01 & 0.821 & 9.871 & $<0.001$ \\
\hline 46 & MIP & $Y=4.04+0.27 X$ & 0.05 & 0.638 & 5.500 & $<0.001$ \\
\hline 46 & $\underline{P}_{\text {PAs }}$ & $Y=5.13+0.09 X$ & 0.02 & 0.535 & 4.213 & $<0.001$ \\
\hline 46 & $\bar{P}_{P A}-P_{L}$ & $Y=7.1+0.09 X$ & 0.05 & 0.254 & 1.740 & $>0.05$ \\
\hline 46 & PVR & $Y=6.39+0.85 X$ & 0.25 & 0.461 & 3.440 & $<0.01$ \\
\hline 38 & $P_{P A d}-P_{L}$ & $Y=5.11+0.06 X$ & 0.10 & 0.106 & 0.639 & $>0.50$ \\
\hline 46 & $\mathrm{P}_{\mathrm{PAs}}-\mathrm{P}_{\mathrm{PAd}}$ & $Y=5.79+0.12 X$ & 0.04 & 0.456 & 3.403 & $<0.01$ \\
\hline \multicolumn{7}{|c|}{ Pulmonary blood volume } \\
\hline 49 & SV & $Y=478.3+1.36 X$ & 0.87 & 0.223 & 1.570 & $>0.10$ \\
\hline 49 & $\mathbf{P}_{\mathrm{L}}$ & $Y=507.0+7.56 X$ & 2.76 & 0.373 & 2.763 & $<0.01$ \\
\hline 49 & TBV & $Y=239.4+0.067 X$ & 0.02 & 0.337 & 2.450 & $<0.02$ \\
\hline 46 & MIP & $Y=472.4+7.09 X$ & 2.35 & 0.425 & 3.125 & $<0.01$ \\
\hline 46 & $\underline{P}_{P A s}$ & $Y=493.5+2.48 X$ & 0.96 & 0.369 & 2.636 & $<0.02$ \\
\hline 46 & $\overline{\mathrm{P}}_{\mathbf{P A}}-\mathrm{P}_{\mathbf{L}}$ & $Y=554.0+2.35 X$ & 1.97 & 0.161 & 1.081 & $>0.20$ \\
\hline 46 & PVR & $Y=556.2+11.73 X$ & 11.14 & 0.160 & 1.074 & $>0.20$ \\
\hline 38 & $P_{P A d}-P_{L}$ & $Y=567.9+2.66 X$ & 3.77 & 0.114 & 0.687 & $>0.40$ \\
\hline 46 & $P_{P A s}-P_{P A d}$ & $Y=520.2+3.11 X$ & 1.56 & 0.294 & 2.042 & $>0.05$ \\
\hline
\end{tabular}

$\mathrm{N}$, number of observations; $\mathrm{S}_{\mathrm{b}}$, standard error of correlation coefficient; $\mathrm{t}_{\mathrm{r}}$, "Student's $t$ " calculated for correlation coefficient; $t=r /\left(1-r^{2} / N-2\right) ; S V$, stroke volume; $P_{L}$, pulmonary venous pressure; $T B V$, total blood volume; MIP, mean intravascular pressure; $P_{P_{A},}$, pulmonary artery systolic pressure; $\mathbf{P}_{\mathbf{P A}_{A}}$, mean pulmonary artery pressure; $\mathrm{P}_{\mathbf{P A d}}$, pulmonary artery diastolic pressure; PVR, pulmonary vascular resistance.

* Simple linear regression equations were calculated by the method of least squares. The dependent variable, $Y$, is either PMTT or PBV, as indicated. The independent variable, $X$, is listed in column labeled "parameter." 


\section{DISCUSSION}

Validation of the method. Of the two methods under discussion, the radiocardiographic method of measuring pulmonary blood volume has the distinct advantage of not requiring left atrial injection or sampling. Hence it can be applied in studies of the pulmonary circulation of patients who would not ordinarily come to left heart catheterization. Nevertheless, this method has not found wide application presumably because the mathematical basis has not been validated by a direct comparison in the same individuals with the double-injection dye technique.

The present report indicates that agreement between the two methods is good over a wide range of transit times. The apparent tendency of PMTT $T_{\text {Rrsa }}$ to exceed PMTT $_{\text {Dye }}$ is not statistically significant. Although individual discrepancies of greater than $25 \%$ were observed in four of the 25 comparisons, this proportion is not greater than that reported by Samet, Bernstein, Lopez, and Levine who compared two dye injection methods of measuring PMTT (27).

The method of analysis of radiocardiograms proposed by Giuntini et al. (14) is based on assumptions regarding size of ventricular chambers and distribution of pulmonary transit times in addition to the usual assumptions underlying the use of indicators to measure the volume of vascular sections. These assumptions may not be valid for every patient, thus giving rise to indeterminate errors in measurement of PBV. In their original publication, from analysis of curves generated by a digital computer incorporating different chamber sizes and transit time distributions, these authors concluded that the magnitude of error between true, i.e. imposed, mean transit time and calculated PMTT would rarely exceed three heart cycles. In fact, in the present comparisons only two of $25 \mathrm{PMTT}_{\mathrm{RISA}}$ values were more than three heart cycles different from PMTT Dye. Assuming PMTT $_{\text {Dye }}$ values to represent true mean transit times, the range of errors of PMTT $T_{\text {RISA }}$ closely approximates the prediction and justifies the simplifying assumptions.

Reproducibility of $\mathrm{PBV}_{\mathrm{RISA}}$ determinations in this laboratory is not substantially different from the experience of several groups measuring pulmonary blood volume. Giuntini, Maseri, and Bianchi (28) report an

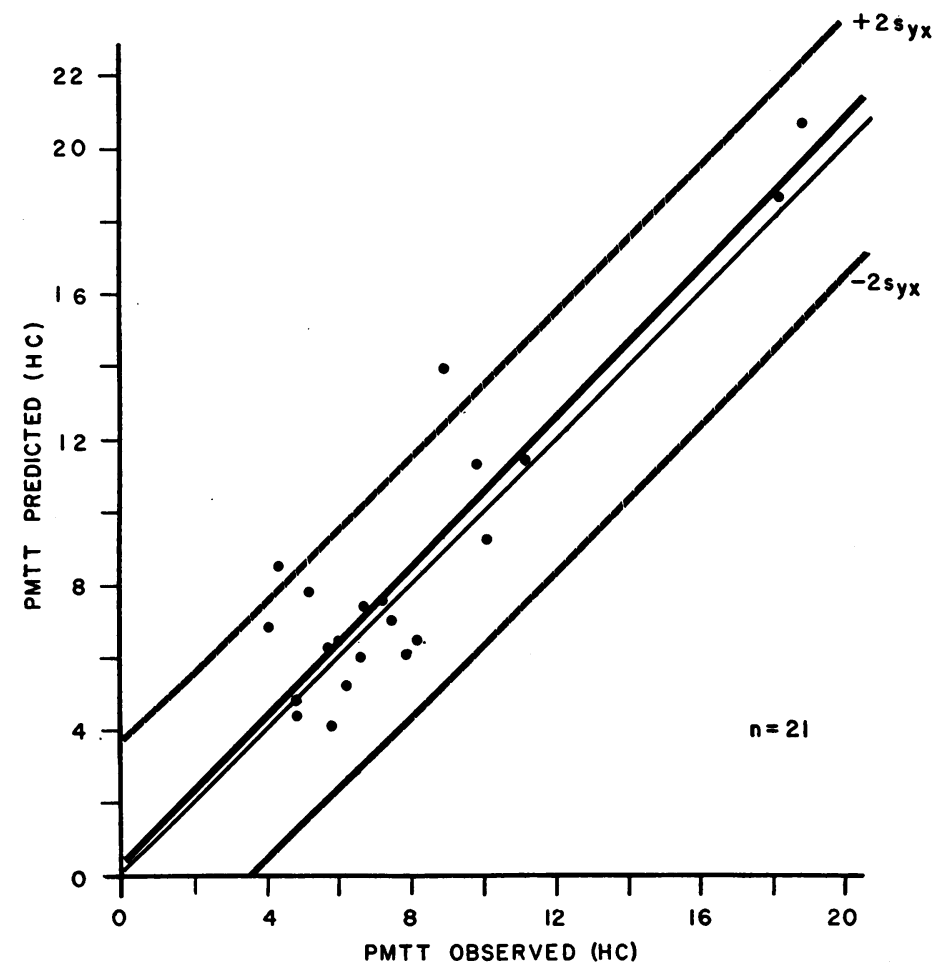

FIGURE 4 Comparison in 21 patients (Group B) of observed and predicted pulmonary mean transit time. PMTT was predicted on the basis of an estimating equation (equation 6 in text) derived from data from a different group of 49 patients (Group A). HC= heart cycle. $s_{y \cdot x}=$ standard error of estimating equation. The heavy solid line is the regression line of predicted on observed values; the fine solid line is the line of identity. The regression line and 19 of 21 points fall within two standard errors of estimate of the line of identity. 


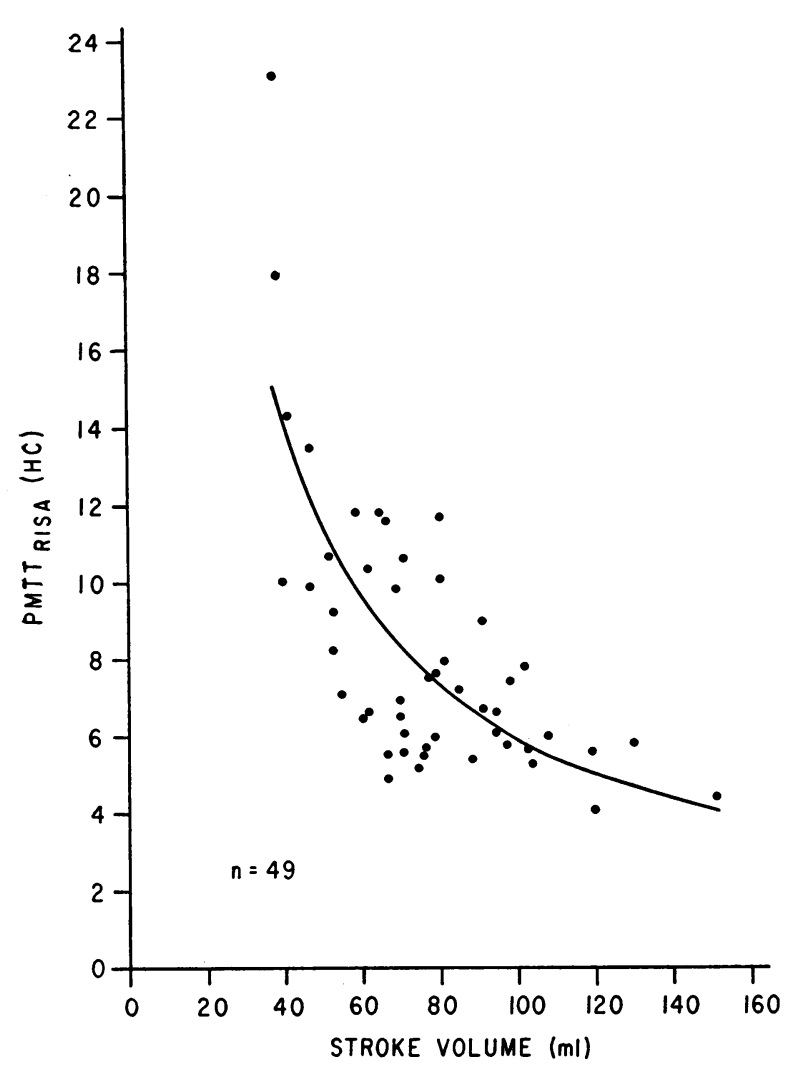

FIGURE 5 Relation of pulmonary mean transit time and stroke volume. The solid line is the regression line, calculated as $\mathrm{PMTT}=K(1 / \mathrm{SV})$.

average variation of $2.7 \%$ about the mean of duplicate values of $P B V_{\text {RIsA, }}$, the greatest difference between duplicates being $15.4 \%$. Data in the present group yields an average variation of $5.0 \pm 2.8 \%$ about the mean of PBV $21.6 \%$. Similar statistics calculated for data published by $\mathrm{Yu}(15)$ result in an average variation of $4.2 \pm 2.1 \%$ about the mean of two PBVDye values in 19 patients; deFreitas, Faraco, Nedel, deAzevedo, and Zaduchliver obtained a value of $3.2 \pm 2.7 \%$ in their patients (11).

Samet et al. (27) in their large series of PBV terminations indicate that $21 \%$ of duplicates (18 of 86 ) were in excess of $20 \%$ different from the first determination, whereas in the present series $6 \%$ of duplicates $(2$ of 34 ) were in excess of $20 \%$ different from the first value. Forsberg chose to compare duplicates by a ratio of first to second determinations, and reported a mean value of $1.00 \pm 0.11(10)$. The mean ratio between 34 duplicate $\mathrm{PMTT}_{\mathrm{RISA}}$ values measured in this laboratory is $0.98 \pm 0.09$.

Since blood flow enters into the calculation of pulmonary blood volume as well as into the statistical analysis of its determinants, the validity of this determination was also evaluated. Cardiac output determination from radiocardiograms has previously been validated by comparison with the Fick method (29). In this laboratory 60 comparisons with Fick determinations again showed no significant difference $(t=1.558,0.2>P>0.1)$. The RCG and dye dilution measurements of flow were also in agreement. In comparison with previously published data, the standard deviation of the ratio $Q_{\text {rrss }}: Q_{\text {Dye }}$ is $18 \%$, similar to the figure of $15 \%$ found by Dock, Krauss, McGuire, Hyland, Haynes, and Dexter when comparing flow values calculated from the simultaneous injection of two indicators into the pulmonary artery and left atrium (3). The standard deviation of differences between $Q_{\mathrm{rrss}}$ and $Q_{\mathrm{Dye}}$ is $718 \mathrm{ml}$, close to the figure of $650 \mathrm{ml}$ obtained by Forsberg (10) utilizing a method similar to that of Dock et al. (3). Duplicate determinations of $Q_{\text {rrss }}$ in this laboratory may be expected to fall within $20 \%$ of each other ( $95 \%$ confidence). Finally, total blood volume determinations as calculated from the dilution of T-1824 dye and of radio-iodinated human serum albumin showed no systemic differences in the patient studied. Hence the values of blood flow and total blood volume, which enter into the statistical analysis to be discussed, can be presumed to be valid.

Determinants of pulmonary blood volume. Previously published analyses relating PBV to other hemodynamic parameters are, in some degree, contradictory. Whereas some authors observed a positive relation between PBV and stroke volume $(1,5,9,10,14)$, this relationship cannot be derived from data published by others (4, 6-8) and a negative relationship between these parameters has been published by yet another group (11). Similarly, whereas a positive correlation between left atrial pressure and PBV has been confirmed by some workers $(3,5,10)$, it is specifically denied by others (9). Contradictory results regarding the effects of pulmonary vascular resistance on PBV have also been published $(1,3-5,10)$.

In contrast, statistical analysis of the data from the present series has resulted in an estimating equation for pulmonary mean transit time, which includes only three independent variables, giving rise to an equation for pulmonary blood volume with only two independent variables. Within the error of the radiocardiographic method, $90 \%$ of the observed variability of resting pulmonary blood volume can be ascribed to variation in pulmonary venous pressure and total blood volume.

The simplicity of these equations may be surprising in view of the fact that none of the basic observations is new. The inverse relationship of PMTT and flow was reported by Doyle, Lee, and Kelley (30), and by Dock et al. (3). It is inherent in the definition of mean transit time through a vascular section which exhibits mixing and stationarity, that is, the time required for a 
volume of fluid equivalent to the contained volume to pass through the section. Thus, for the lungs:

$$
\mathrm{PMTT}=\frac{\mathrm{PBV}}{\mathrm{SV}}
$$

Since in the present series the relationship of PMTT and $1 / \mathrm{SV}$ remained statistically significant despite more than twofold differences in PBV, it is apparent that variations in transit time are mediated more by variations in blood flow than by variations in lung blood volume.

An increase in pulmonary blood volume with increasing pulmonary venous pressure was observed by Carlill and Duke (31) and by Sarnoff and Berglund (32) in animal preparations. In man a similar observation was made by Dock et al., (3) McGaff, Roveti, Glassman, and Milnor (5), and by Forsberg (10). The findings in the present series of patients confirm these reports. A graph relating PMTT to $P_{L}$ in these 49 cases actually has the apperance of a parabola, convex to the pressure axis, indicating that below a certain level $P_{L}$ has little effect on prolonging PMTT. However, a multiple regression equation parabolic with respect to $P_{L}$ resulted in an explained variance of PMTT identical to that provided by equation 8 which is linear with respect to $P_{\mathrm{L}}$. Hence, a linear equation adequately describes the statistical relationship between these two parameters.

Several authors $(3,14,15)$ have remarked on a relation between pulmonary blood volume and total blood volume as observed in the present data. However, the major contribution which variations in total blood volume make toward explaining variations in pulmonary blood volume has not been fully appreciated. This may be due to the fact that, although many normal studies have been reported in the literature using the double injection method, there has been little indication for left atrial catheterization in patients with hypervolemia unrelated to heart disease or in patients with primary lung disease. Such studies have been conducted in this laboratory using the RCG method.

Resistance to expansion of pulmonary blood volume has been observed during the administration of plasma expanders but this is presumably due to the rapidity with which the changes are induced $(28,33)$. In the present series where the increase in total blood volume has come about gradually at some antecedent time and where a steady state is under observation, TBV appears to be the primary determinant of PBV.

The conclusion that only variations in SV, TBV, and $P_{L}$ need be invoked in order to explain variations in PMTT observed between patients in the present study is inherent in the highly significant correlation between PMTT and these three parameters and in the reproducibility of the RCG technique. Equation 8 explains
$75 \%$ of the variation of PMTT with a standard error of estimate of 1.8 heart cycles. Since the standard deviation of duplicate PMTT $T_{\text {riss }}$ measurements is 1.1 heart cycles which constitutes $60 \%$ of the error of estimate of equation 8 , then at least $60 \%$ of the residual unexplained variance of PMTT in these 49 cases may be ascribed to random errors of the method.

This in effect leaves only $10 \%$ residual variance of PMTT justifying the use of an estimating equation which passes through the origin and allows no determinants of PMTT other than the three discussed. This does not preclude that some of the observed variation is in fact due to variation in other hemodynamic parameters but the effect must be small.

An explanation for observed variability of PBV has been sought in differences of vascular distensibility by relating $\mathrm{PBV}$ to indices such as pulmonary vascular resistance (PVR) and mean intravascular pressure (MIP). Although each of these factors and several other pressure parameters in the lesser circulation correlate with PMTT in a simple linear regression, none of them produced a significant reduction of variance of PMTT beyond that produced by $\mathrm{SV}, \mathrm{P}_{\mathrm{L}}$, and TBV. Because of the intimate relationship between flow, volume, and transit time, the analyses apply equally to PMTT and PBV; moreover, the conclusions have been confirmed by separate calculations of multiple regression equations for PBV.

Certain authors have found the linear relationship of $\mathrm{PBV}$ and pulmonary venous pressure to be modified in the presence of increased vascular resistance secondary to long-standing mitral stenosis $(3,5)$. As illustrated in Fig. 6, no effect of resistance on the relationship of pulmonary blood volume and venous pressure was observed in the present study. These 49 patients include relatively few with mitral stenosis and high resistance; hence the difference between the present and previously published series may lie in the type of patients evaluated.

Alternatively, the difference may lie in the interpretation of data. Elevated vascular resistance and lower than normal pulmonary blood volume are usually considered to be cause and effect. However, the reverse may be true. An elevated pulmonary blood volume must be accommodated in larger or more numerous channels. If the total cross sectional area of vascular channels in the lung is increased, the gradient of pressure across the lungs for any given flow rate will be lower, i.e., a larger pulmonary blood volume will result in a lower pulmonary vascular resistance.

At least some of the differences in pulmonary blood volume reported in patients with elevated left atrial pressure and pulmonary vascular resistance may be explained by differences in total blood volume. The failure 


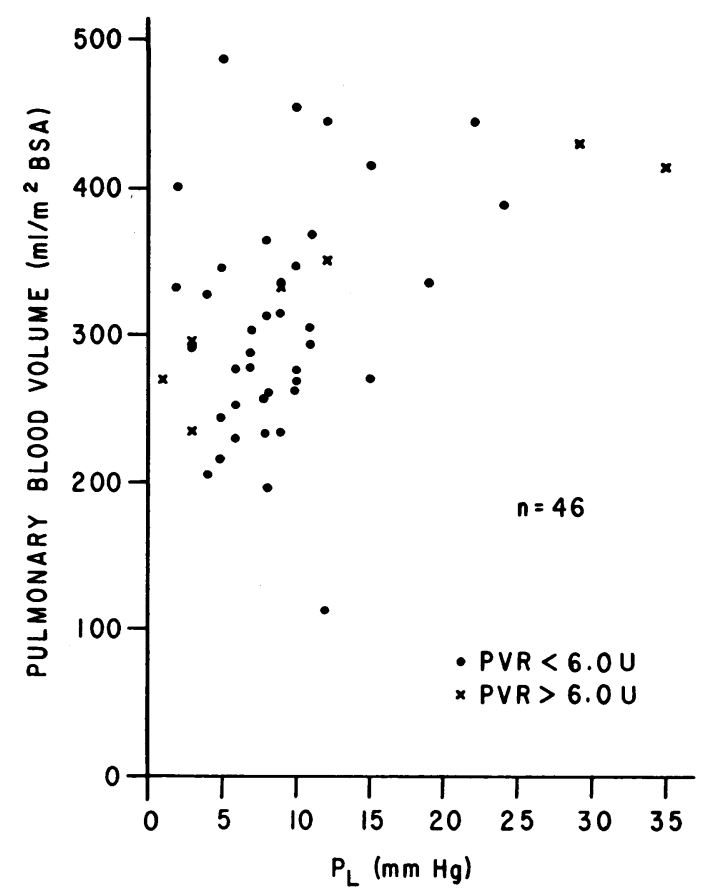

FIgURE 6 Relation of pulmonary blood volume and pulmonary "venous" pressure, $P_{L}$, in 46 patients of Group A. Crosses indicate seven patients with pulmonary vascular resistance (PVR) greater than $6.0 \mathrm{U},[\mathrm{U}=(\mathrm{mm} \mathrm{Hg}) /$ (liters) $\min$ per $\left.\left.\mathrm{m}^{2} \mathrm{BSA}\right)\right]$. For discussion see text.

to specifically consider this factor as a determinant of $P B V$, and the small residual variance left unexplained by $P_{L}$ and TBV may account for the contradictory results obtained by other workers. Since data on total blood volume have not been published in previous reports, this possibility cannot be evaluated.

Also contrary to published work is the absence of a significant regression of $\mathrm{PBV}$ and $\mathrm{SV}$ in Group $\mathrm{A}$ when these 49 patients were considered as a single group. If the highest values of PBV were eliminated by omitting data from patients with elevated pulmonary venous pressure ( $>12 \mathrm{~mm}$ of $\mathrm{Hg}$ ) and those with elevated total blood volume ( $>3.1$ liters $/ \mathrm{m}^{2}$ BSA), a significant regression was observed in the remaining 28 patients. Of these 28, 13 exhibited an elevated PBV $(>300 \mathrm{ml} /$ $\left.\mathrm{m}^{2} \mathrm{BSA}\right)$; however, stroke volume was not elevated in these 13 patients being equally distributed about the mean SV for the group of 28 . In contrast, total blood volume was elevated above the mean in 10 of the $13 \mathrm{pa}$ tients with abnormally high pulmonary blood volume. This suggests that in these 13 patients also pulmonary blood volume paralleled total blood volume, and the correlation of $\mathrm{PBV}$ and $\mathrm{SV}$ was due in large part to the tendency of stroke volume to increase with total blood volume in the absence of myocardial insufficiency.
The implication of the derived estimating equation for $\mathrm{PBV}$, which is linear with respect to pulmonary venous pressure and total blood volume, is that variations in resting pulmonary blood volume are the result of passive distention of the pulmonary bed. With the possible exception of patients with increased pulmonary vascular resistance secondary to mitral stenosis, who were not adequately represented in this series, no evidence of altered vascular distensibility was found in the 70 patients (Groups A and B) studied. From this data it appears that the pulmonary blood volume normally constitutes a rather fixed fraction of total blood volume, the proportion being changed only passively, by an increase in outflow pressure from the pulmonary bed. This concept has been voiced before but has not been adequately quantitated. It is clear that since $90 \%$ of the variability of resting pulmonary blood volume can be explained by variation in these two factors the residual effects of other factors, such as vascular compliance, must be small and hence difficult to quantitate and of doubtful significance.

\section{REFERENCES}

1. Milnor, W. R., A. D. Jose, and C. J. McGaff. 1960. Pulmonary vascular volume, resistance and compliance in man. Circulation. 22: 130.

2. Fujimoto, K., R. Kunieda, and T. Shiba. 1960. Lung blood volume in acquired valvular disease. Jap. Heart J. 1: 442 .

3. Dock, D. S., W. L. Kraus, L. B. McGuire, J. W. Hyland, F. W. Haynes, and L. Dexter. 1961. The pulmonary blood volume in man. J. Clin. Invest. 40: 317.

4. Oakley, C., G. Glick, M. N. Luria, B. F. Schreiner, and P. N. Yu 1962. Some regulatory mechanisms of the human pulmonary vascular bed. Circulation. 26: 917.

5. McGaff, C. J., G. C. Roveti, E. Glassman, and W. R. Milnor. 1963. The pulmonary blood volume in rheumatic heart disease and its alteration by isoproterenol. Circulation. $27: 77$.

6. Yu, P. N., G. Glick, B. F. Schreiner, and G. W. Murphy. 1963. Effects of acute hypoxia on the pulmonary vascular bed of patients with acquired heart disease. Circulation. 27 : 541.

7. Glick, G., B. F. Schreiner, G. W. Murphy, and P. N. Yu. 1963. Effects of inhalation of 100 per cent oxygen on the pulmonary blood volume in patients with organic heart disease. Circulation. 27: 554.

8. Schreiner, B. F., G. W. Murphy, G. Glick, and P. N. Yu. 1963. Effect of exercise on the pulmonary blood volume in patients with acquired heart disease. Circulation. 27: 559.

9. Vernauskas, E., S. A. Forsberg, J. Widimsky, and S. Paulin. 1963. Pulmonary blood volume and its relation to pulmonary hemodynamics in cardiac patients. Acta Med. Scand. 173: 529.

10. Forsberg, S. A. 1964. Pulmonary blood volume in man. Acta Med. Scand. 175 (Suppl) : 410.

11. deFreitas. F. M., E. Z. Faraco, N. Nedel, D. F. deAzevedo, and J. Zaduchliver. 1964. Determination of pulmonary blood volume by single intravenous injection of 
one indicator in patients with normal and high pulmonary vascular pressures. Circulation. 30: 370.

12. Samet, P., W. H. Bernstein, A. Medow, and S. Levine. 1965. Transseptal left heart dynamics in thirty-two normal subjects. Dis. Chest. 47: 632.

13. Donato, L., C. Giuntini, M. L. Lewis, J. Durand, D. F. Rochester, R. M. Harvey, and A. Cournand. 1962. Quantitative radiocardiography. I. Theoretical considerations. Circulation. 26: 174.

14. Giuntini, C., M. L. Lewis, A. Sales Luis, and R. M. Harvey. 1963. A study of the pulmonary blood volume in man by quantitative radiocardiography. J. Clin. Invest. 42: 1589.

15. Yu, P. N. 1969. Pulmonary Blood Volume in Health and Disease. Lea \& Febiger, Philadelphia.

16. Ross, J., Jr., E. Braunwald, and A. G. Morrow. 1960. Left heart catheterization by the transseptal route. A description of the technic and its applications. Circulation. 22: 927.

17. Seldinger, S. I. 1953. Catheter replacement of the needle in percutaneous arteriography. Acta Radiol. 39: 368.

18. Kaltman, A. J., W. H. Herbert, R. J. Conroy, and C. E. Kossmann. 1966. The gradient in pressure across the pulmonary vascular bed in diastole. Circulation. 34: 377.

19. Donato, L., D. F. Rochester, M. L. Lewis, J. Durand, J. O. Parker, and R. M. Harvey. 1962. Quantitative radiocardiography. II. Technic and analysis of curves. Circulation. 26: 183.

20. Veall, N., J. D. Pearson, T. Hanley, and A. E. Lowe. 1.954. A method for the determination of cardiac output (preliminary report). In Proceedings of the Second Radioisotope Conference, Oxford. Butterworths Scientific and Medical Publications, London. Vol. 1. 183.

21. Hamilton, W. F., J. W. Moore, J. M. Kinsman, and R. G. Spurling. 1932. Studies on the circulation. IV. Further analysis of the injection method, and of changes in hemodynamics under physiological and pathological conditions. Amer. J. Physiol. 99: 534.

22. McNeely, W. F., and M. A. Gravellese. 1954. Measure- ment of cardiac output by dye dilution technique: use of an "integrated" sample collection in calibration of the photometric instrument. J. Appl. Physiol. 7: 55.

23. Chaplin, H., Jr., and P. L. Mollison. 1952. Correction for plasma trapped in the red cell column of the hematocrit. Blood. $7: 1227$.

24. Fudenberg, H., M. Baldini, J. P. Mahoney, and W. Dameshek. 1961. The body hematocrit/venous hematocrit ratio and the "splenic reservoir." Blood. 17: 71.

25. Steel, R. G. D., and J. H. Torrie. 1960. Principles and Procedures of Statistics. McGraw-Hill Book Company, New York.

26. Ezekiel, M. J. B., and K. A. Fox. 1959. Methods of Correlation and Regression Analysis. John Wiley \& Sons, Inc. New York.

27. Samet, P., W. H. Bernstein, A. Lopez, and S. Levine. 1966. Methodology of true pulmonary blood volume determination. Circulation. 33: 847.

28. Giuntini, C., A. Maseri, and R. Bianchi. 1966. Pulmonary vascular distensibility and lung compliance as modified by dextran infusion and subsequent atropine injection in normal subjects. J. Clin. Invest. 45: 1770.

29. Lewis, M. L., C. Giuntini, L. Donato, R. M. Harvey, and A. Cournand. 1962. Quantitative radiocardiography. III. Results and validation of theory and method. Circulation. 26: 189.

30. Doyle, J. T., R. A. Lee, and E. B. Kelley. 1952. Observations on the elasticity of the pulmonary vasculature in man. Amer. Heart J. 44: 565.

31. Carlill, S. D., and H. N. Duke. 1956. Pulmonary vascular changes in response to variations in left auricular pressure. J. Physiol. (London). 133: 275.

32. Sarnoff, S. J., and E. Berglund. 1952. Pressure-volume characteristics and stress relaxation in the pulmonary vascular bed of the dog. Amer. J. Physiol. 171: 238.

33. deFreitas, F. M., E. Z. Faraco, D. F. deAzevedo, J. Zaduchliver, and I. Lewin. 1965. Behavior of normal pulmonary circulation during changes of total blood volume in man. J. Clin. Invest. 44: 366. 